Outcomes of a Student Research Project on Circular Building Systems Focus on the Educational Aspect

Peer-reviewed author version

JANSSENS, Bart; KNAPEN, Elke; WINKELS, Peggy \& VERBEECK, Griet (2019) Outcomes of a Student Research Project on Circular Building Systems - Focus on the Educational Aspect. In: Transition Towards a Net Zero Carbon Built Environment, 323 (Art № 012138)..

DOI: $10.1088 / 1755-1315 / 323 / 1 / 012138$

Handle: http://hdl.handle.net/1942/29044 


\title{
Outcomes of a Student Research Project on Circular Building Systems - Focus on the Educational Aspect
}

\author{
Janssens B., Knapen E., Winkels P., Verbeeck G. \\ Faculty of Architecture and Arts, Hasselt University, Diepenbeek, Belgium \\ bart.janssens@uhasselt.be
}

\begin{abstract}
The growing need to shift from a linear to a circular economy has inspired producers of building materials and systems to innovate their products to match the requirements of a future circular economy. Others have been developing modular systems in the past to simplify the building process and are now reconsidering these products for their potential in a circular economy. However, at this early stage on the road towards a circular economy, claims of circularity are often made too easily and many producers as well as architects and builders are still struggling with the practice of circular building. In this context and within an assignment for master students in architecture, three cavity walls, each $9 \mathrm{~m}^{2}$ and composed with a different system for the inner and the outer wall, have been constructed and disassembled in order to test their potential for circularity. The extent of circularity has been critically analysed with an existing framework of evaluation criteria for design for change: three criteria on interface level (reversibility, simplicity, speed), three on component level (compatibility, durability, manageability) and three on composition level (independence, pace-layering, prefabrication). This analysis was complemented with an environmental assessment of the materials used and with interviews with contractors who have experience with these systems. In a final step, proposals for improvement of the products and systems have been made and tested on a mockup scale, in order to better fit a possible future circular economy.
\end{abstract}

\section{Introduction}

The exploitation of resources and the linear way in which materials are harvested, consumed and discarded cannot be sustained anymore. The current economy must be transformed to a circular economy model in which a careful use of resources is part of managing a sustainable development. Strategies on an institutional level [1] (e.g. Flemish policy action 'Vlaanderen Circulair': https://www.vlaanderen.be/nl/vlaamse-regering/transitie-circulaire-economie), indicators and research projects have been initiated. Circularity in the building sector is high on public (nationally and internationally) and corporate agendas. As circular building will become key in the professional field, it is necessary that architecture students are being prepared.

Incorporation of circular principles has only begun recently. Producers of building materials and construction systems are rapidly taking the first steps and develop or redevelop products in view of the requirements for circular building. However, the risk is high that in the rush to quickly develop products, claims of circularity are made too easily. 
Within this context, master students in architecture of the Faculty of Architecture and Arts of Hasselt University studied the potential for circular building of specific building systems. The objectives were threefold, in order of importance: educational, research methodological, niche development. The educational aspects included i.a.: trigger and train students to become architect-designers with a strong focus on circularity; and the competence to conduct research in a collaborative and critical way. The research methodological objectives consisted of exploring possibilities for actively involving architecture students in research on building-technical aspects (the nexus education - research). The niche development objective fits the research field of the research line 'Sustainability' within the Research Group Arck of the faculty, framed within the 'civic university' model adopted by Hasselt University. Research is conducted on building-technical niches with a societal relevance, in this case building systems that enable circular building and enhance a circular economy model.

Primary aim of this paper is to present and discuss the concept of the student research. Secondary aim is to present tentative and illustrative outcomes of selected circular building systems.

After this first section, this paper consists of four more sections. The second section provides a description of the concept of the student assignment. The third section presents selected systems and composed cavity wall ensembles for the student research. In the fourth section, illustrative results are given and discussed on both the concept of the research as on selected systems and composed ensembles. Finally, a conclusion wraps up findings and perspectives.

\section{Concept of the research}

\subsection{Description of the assignment}

2.1.1. Positioning within the curriculum. The master programme of architecture at Hasselt University includes elective research seminars in which the nexus 'education - research' is operationalized. Each of the four defined seminars is linked to a research line of the research group Arck. The seminar 'Building concept' is substantiated by the research line 'Sustainability' of which the research is closely linked to the growing need to deal with the built environment in a more sustainable way. The objectives of 'Sustainability' are (1) to contribute to the scientific founding of sustainable architecture mainly at building level and with a focus on comfort, energy and material performance, and (2) to contribute to supporting the decision making process of both architects and building owners towards more sustainable designs, in line with the ambitions of the EU and Flanders for nearly zero energy buildings and more sustainable material use.

As from the academic year 2017 - 2018 onwards, the seminar 'Building concept' focuses on 'designing and building for/from circularity'. In a living-lab set-up, theory and experiment are used by students, as both designers and builders, to explore the concept of 'circularity' in the built environment. The focus alternates from year to year: one year theoretical models for circularity are studied by research by design on the building level; in the next year, specified circular building systems on the element level are tested. A constant throughout the two years is a critical analysis, a reflection and an optimization.

2.1.2. The assignment as such. Framed within the second part of the two-year cycle, academic year 2018 - 2019 focused on in-situ testing and improving circular building systems for cavity walls. Characteristic for cavity walls are a layered composition of an inside wall (mostly brick), thermal insulation, a narrow cavity, and a façade (mostly fancy brickwork) [2].

In three groups of each five students (mix of first and second master students), the aim was to test the potential of six preselected systems for circularity by the act of building, backed by an assessment with 
an existing framework of evaluation criteria for design for change and with an existing eco-design tool to evaluate and optimize the environmental impact of the materials used.

The assignment evolves from hands-on explorations, over analysis and assessment by desk-top research, to the formulation of improvements by design \& build research. The research assignment consisted of six phases. In the first phase, manufacturers of selected systems presented their products to the students. In the second phase, cavity wall compositions were developed by using two circular building systems (one for the inner leaf; another for the outer leaf). During the third phase, students had to detail the building knots (scale 1:20 to 1:5) of a given in-situ building experiment. The act of building, build and disassemble, was operationalized in phase four. In overlap with phase four, phase five consisted of an assessment. In the sixth and final phase, possible improvements, material wise and/or building-technical wise, were explored and tested in mock ups.

2.1.3. Learning objectives. The set-up of the research seminar holds three learning objectives. A first, and most obvious, objective is to trigger and train students to become (better) circular-thinking architectdesigners and builders. The two other objectives, explained in paragraphs below, have a more generic feature: learning and preparing to work in a collaborative way; and the development of critical thinking skills.

In recent years, focus is laid on an 'Integrated Design Process' (IDP). An IDP is a process that purposefully brings together the work of various design and engineering disciplines in order to achieve successful projects outcomes. Following Rovers [3], the IDP is characterized by the integration of several activities in a project team. During an entire project development process, several disciplines/stakeholders (the client, the architect-designer, the specialist-engineers), or the Integrated Design Team (IDT), are involved. The IDP implies a social process between the members of the IDT. "Projects participants work and make decisions not in isolation, but rather within a framework of social interaction". One of the key features of an IDP is working in a group. Due to the growing degree of specialisation of the aspect of designing and building this collaborative way is urged [3-4-5], and therefore seen as an important learning objective.

To prepare students for the complex and dynamic world of the 21 st century, professionally, socially and personally, it is necessary to develop critical thinking skills. This is a core theme in contemporary education made explicit by the World Economic Forum [6]. Critical thinking helps to: make informed decisions without being biased; handle, deal with the abundance of information available; understand the learning material and to use it in different contexts; continue learning; deliver a respectful and rational contribution to society; support the transition from the study time to the professional life.

\subsection{Framework of evaluation criteria for design for change}

For the fifth phase of the assignment, the assessment, the design guidelines for 'design for change' on the element level developed by the Flemish agency for waste management (OVAM) based on Vandenbroucke [7] were used. Nine criteria are differentiated in three aspects: interface, which focuses on the interaction between components, holds the criteria 'reversibility', 'simplicity', and 'speed'; components, which focuses on the features of the parts, holds the criteria 'compatibility', 'durability', and 'manageability'; and composition, which focuses on the whole, holds the criteria 'independence', 'pace-layering', and 'prefabrication'.

\subsection{Complementary assessment}

In order to have a more complete and a non-univocable evaluation, the environmental impact of selected building systems was assessed with the Ecolizer tool (http://www.ecolizer.be/), an eco-design tool for product design made available by OVAM.

By initiation, complementary assessment was targeted with interviews with contractors who have experience with the systems. However, this was canceled due to time restrictions. 


\section{Selected systems, composed cavity wall ensembles and set-up building experiment}

\subsection{Systems}

Six manufacturers were willing to collaborate in the research: system 1 - Construclick; system $2-$ Facadeclick; system 3 - Systimber; system 4 - Clickbrick; system 5 - Steko; system 6 - Facatile.

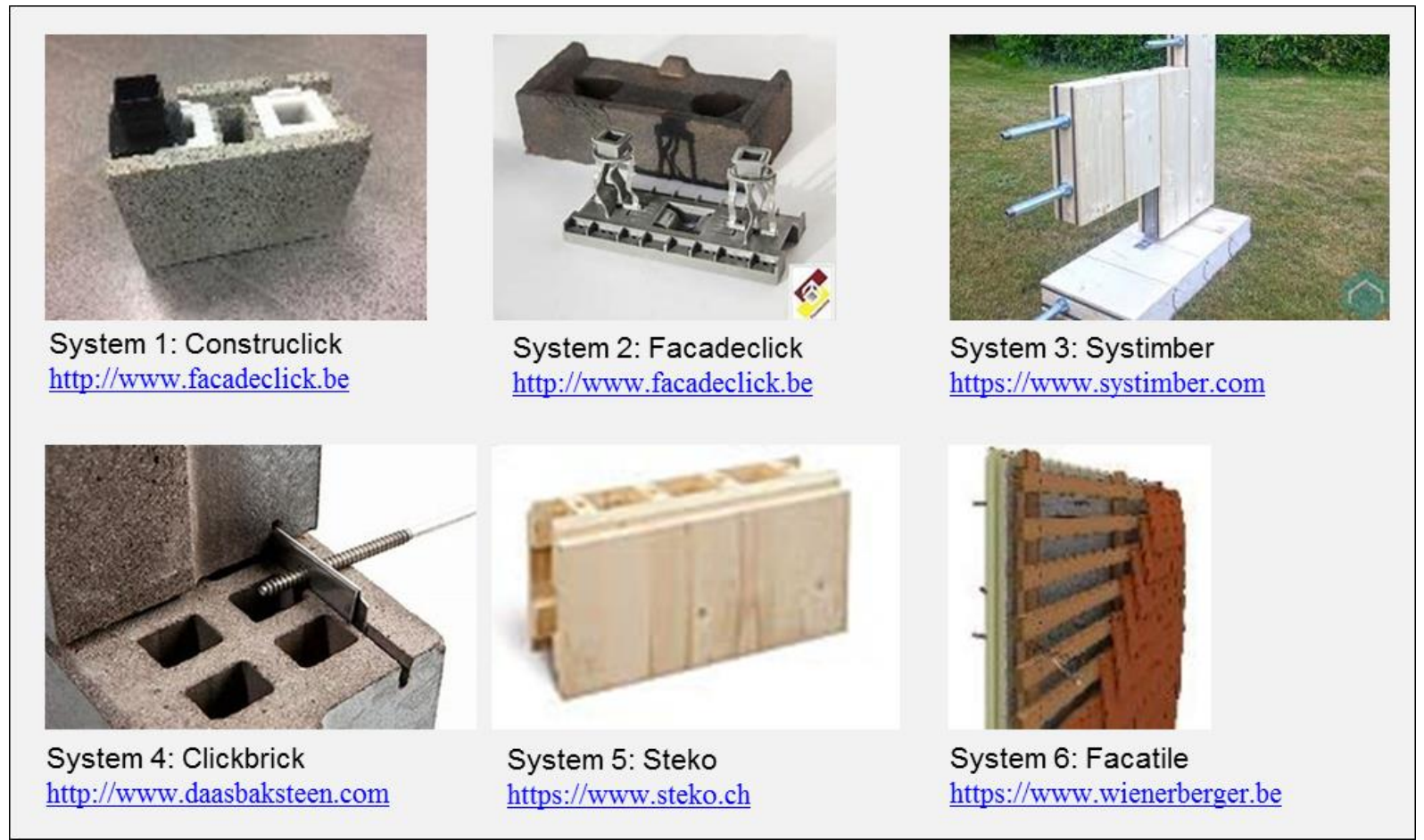

Figure 1. The six building systems selected.

\subsection{Compositions}

Focused on the aspect of cavity walls and taking into account the availability of testing materials, two cavity wall compositions were retained by the tutors, and detailed by the students: composition 1 Construclick/Facadeclick; composition 2 - Systimber/Clickbrick (see figure 2).

\subsection{Set-up building experiment}

The in-situ building experiment was conducted at the faculty. Framed within an existing floor, concrete columns and beams, two set-ups of cavity wall constructions of $9 \mathrm{~m}^{2}$ including a window (A, with a corner; B, without a corner) were designed, built and disassembled (see figure 2).

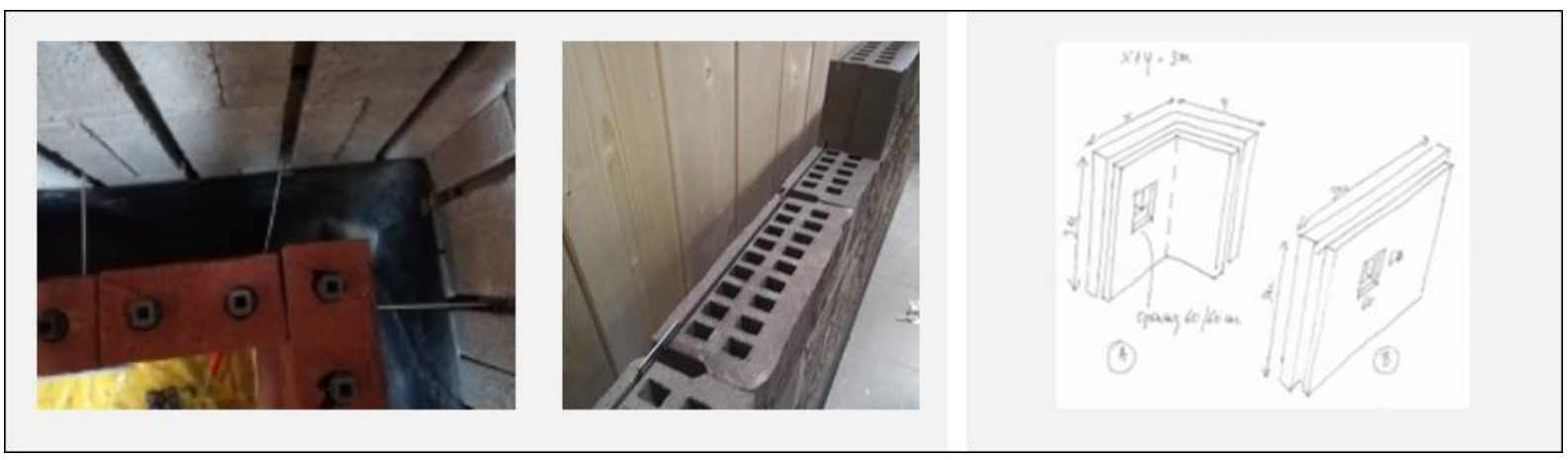

Figure 2. Left, the two compositions $1 \& 2$; right, the two set-up building experiments A \& B. 


\section{Results and discussion}

Following outlined objectives, this section provides and discusses results: first, regarding the educational and research set-up; second, regarding the studied systems and compositions. The objectives were three-fold, in order of importance: educational, research methodological, niche development.

\subsection{Educational aspects}

4.1.1. Students' perspective. After the research, students had to submit a self and peer assessment, including a personal reflection on the assignment. These submissions provided valuable insights in the students' perspective on the seminar.

The in-depth focus on materials and construction methods, and having the time and space to experiment with details of/for new ways of building, was believed to be an added value for the curriculum. The setup of the seminar, with its structure and balance between theory and practice, enabled to gain good insights in circular building, regarding the challenges and opportunities, both for architects and product developers. In addition, the seminar enabled to develop critical thinking skills, inter alia by including an assessment tool. This assessment tool appeared to be both a blessing and a curse, as it was found to be time consuming.

Students appreciated the differing learning process than they are used to. Especially the practice-based approach with the hands-on experimenting was interesting and helpful to gain insights into circular design aspects. In fact, students state that, although it requires a lot of work, more hands-on learning activities in the curriculum would be welcomed. To strengthen their vision, suggestion was made to complement the assignment with a real-life full design/build project such as a small pavilion or a tiny house. This way, students could go beyond analysing materials/products/systems and use their creativity not only as builders but also as designers of spaces and places.

The peer assessment showed that the necessity of working in groups had both positive and negative aspects. Positive was the fact that students with other abilities and points of interest can work together, strengthen the quality of work and learn from each other. Negative part was that working in a team could be difficult, especially when students do not know each other well, when students only see their own opinion, or when students do not show interest or do not collaborate.

4.1.2. Tutors' perspective. Despite the labour intensive preparation, both the collaboration/interaction with system manufacturers and the hands-on approach were seen as promising for the research seminar and will be retained for and even elaborated in following editions. Three issues that must be addressed and need improvement are: (1) the aspect of working in group by students; (2) the aspect of (learning to) assess(ing) in an objective way; and (3) the aspect of encouraging/stimulating/feeding innovation during the development of improvements by the tutors.

4.2. Research methodological aspects in view of the nexus education - research. The nexus worked well mainly due to three aspects. First, the research topic is linked with the architecture and building practice, and has a strong societal relevance. Second, used research methods include tangible aspects (hands-on), and enable to expose outcomes (visibility). Both aspects enable to engage students more easily. Third, an assessment tool is included in the assignment, so outcomes can be validated and incorporated in scientific/academic research. Regarding the latter, a quantitative assessment method is preferred in order to avoid subjective assessments. 


\subsection{Niche development aspects}

For each of the systems and compositions, students proposed and developed improvements based on issues identified by the assessment conducted. This subsection provides some illustrative proposed improvements.

4.3.1. Systems $1 \& 2$ - composition A. For systems 1 (Construclick) and 2 (Facadeclick) as a whole, three suggested improvements are discussed: a) a circular connection of the systems with the foundation; b) a circular fixation of the watertight slab in connection with the foundation; c) an alternative cavity anchor (see figure 3 ).

Within the 'reversible' criterion, the assessment showed that the connection with the foundation of both the inner and outer leaf of the composition, respectively system 1 - Construclick and system 2 Facadeclick, does not fit the idea of circularity. The application guidelines of both systems prescribe the use of a mortar layer. Students experimented with a mounting lath on a levelled basis (foundation).

The watertight connection between the foundation and the inner leaf is not 'reversible', at least not in the sense of reusing materials. Normally the watertight slab is glued on the outer side of the inner leaf. In order to eliminate this gluing, students thought of a connecting block which can be inserted in the gloves of the inner wall (to fix the cavity anchors). By means of rubber washers and metal screws the watertight barrier can be fixed to this connecting block.

Within the assessment criterion 'simplicity' and 'durability', the used cavity anchors demonstrated weaknesses. The form of the metal anchors needed some adjustments (bending) in order to be placed correctly in the spacers of the inner and outer leaf. This bending was also needed during disassembly so cavity anchors showed usage damage. To tackle this, students designed a compressible anchor which facilitates an easy placement and prevents usage damage.

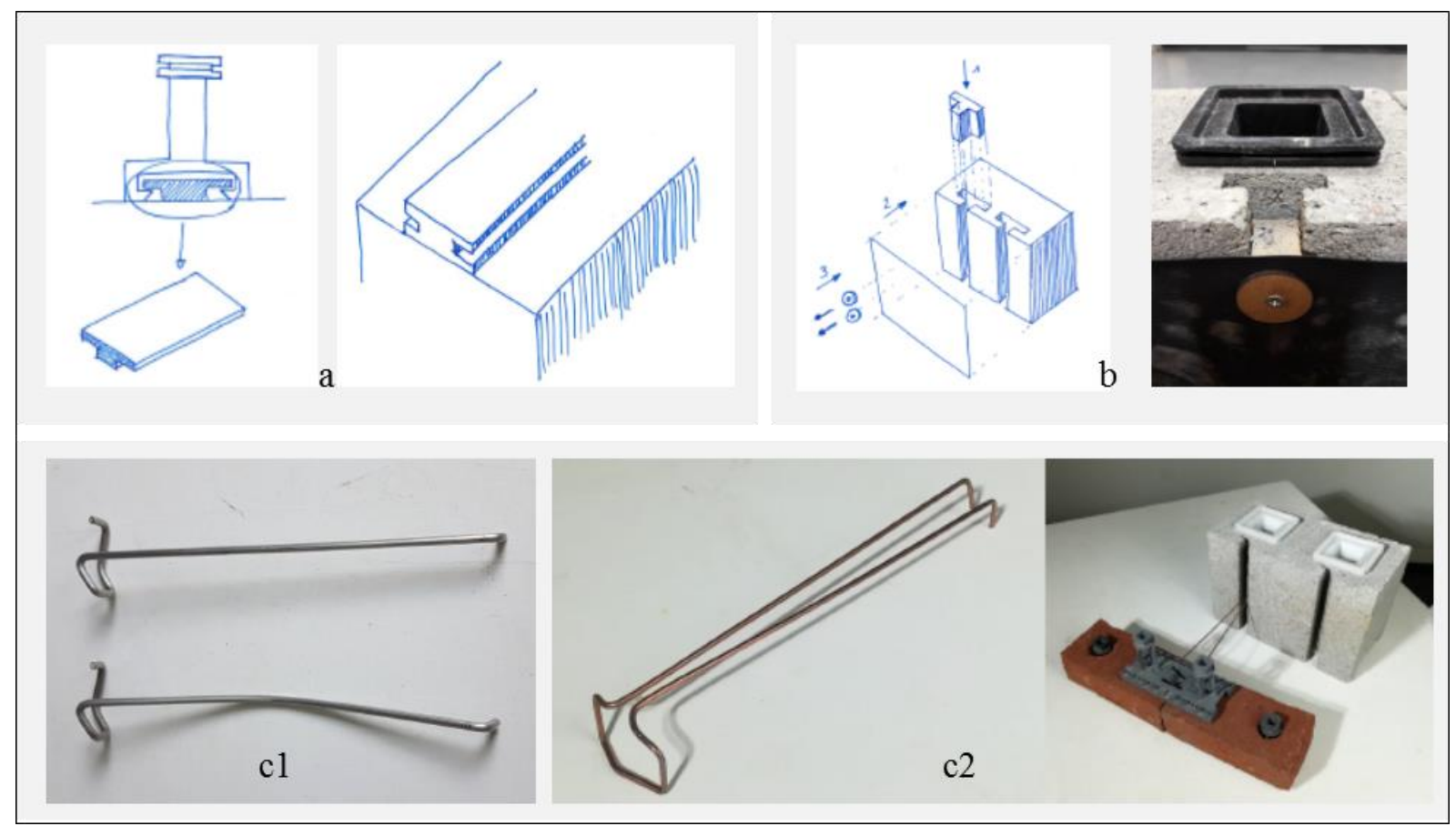

Figure 3. Three suggested improvements for systems $1 \& 2$ - composition A: a) a mounting lath for a circular connection with the foundation; b) a connecting block to fix the watertight slab between foundation and inner wall; c) with $\mathrm{c} 1$ the existing cavity anchor, and $\mathrm{c} 2$ an alternative cavity anchor. 
4.3.2. Systems $3 \& 4$ - composition B. For both systems, Systimber (system 3 ) and Clickbrick (system 4), two suggested improvements are discussed (see figure 4).

During disassembly, it became clear that the metal spacers to connect the wooden beams of the Systimber system were not 'reversible'. Due to a limited grip of the screw machine cap on the head of the spacer, spacers showed such damage that reuse is impossible. Students experimented with wider grip surfaces of the head of the spacer.

Within a cavity-wall composition, Systimber is only limited circular as cavity anchors are drilled in and leave boreholes after disassembly. This may limit the potential for reuse. To prevent this, students designed two possibilities to anchor cavity anchors without leaving traces: first, a slidable two-part connector, in which one part is fixed permanently on the wood and where on the other part the cavity anchor is fixed; and second, the integration of a U-shaped profile in the wood with vertically slidable hooks on which cavity anchors are fixed.

Similar as in systems $1 \& 2$, the Clickbrick system lacks a circular connection with the foundation. Taking into account the specificities of the system, students designed a metal plate which must be screwed on a levelled basis (foundation).

Regarding the cavity anchors included in the Clickbrick system, a problem of 'reversibility' may appear as it is difficult to unscrew them. Students propose to provide the anchors with a screw head so they can always be screwed out easily.

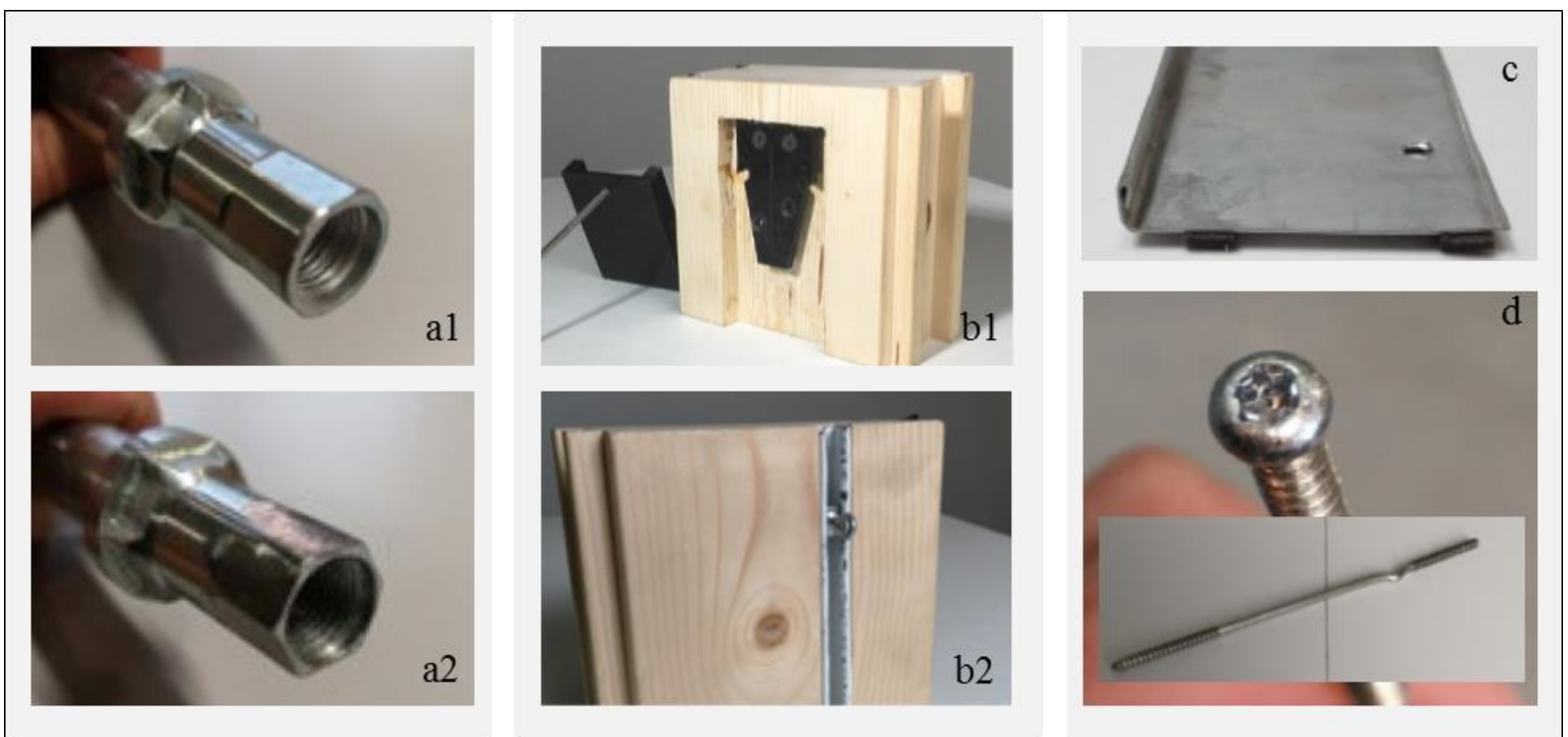

Figure 4. Three suggested improvements for systems $3 \& 4$ - composition B: a) wider grip surfaces of the spacer (with a1 the existing grip); b1 + b2) two anchoring solutions for cavity anchors; c) metal plate for a circular connection with the foundation; d) a screw head of the cavity anchors.

\section{Conclusion}

This paper presented and discussed the concept of the student research on in-situ testing and improving of circular building systems for cavity walls within the context of the research seminar 'Building concept' of the architecture programme of Hasselt University.

Based on reflections of both students and tutors, it can be concluded that the concept of the nexus education - research was successful and only needs minor revisions. Students appreciated the focus on 
new ways of building with a strong societal relevance, the balance between theory and practice and the hands-on experimenting. From the tutors' perspective, the collaboration/interaction with system manufacturers and the hands-on approach was evaluated positive.

Perspectives for future editions of similar student research are: regarding the topic, complementing the assignment with a real-life full design/build project; regarding the educational perspective, including a team building activity in order to improve the aspect of working in a group, and using tailored pedagogical methods to encourage/stimulate/feed innovation during the development of improvements by the students; from a research point of view, selecting a quantitative assessment method which avoids subjective assessments, and which is easy and quick to use by students.

Concerning the circular building systems, the research demonstrated that all systems as such work, are easy to use and fast to assemble and disassemble. Small-scale incremental improvements, as proposed by the students, on the individual system level are required. However, fundamental improvements and research regarding following aspects (non-exhaustive) are believed to be crucial for a full and successful application of the idea of circular building: circular connections between circular building systems and other building components (e.g. foundation, windows, roofs), compatibility (e.g. dimensions, connections) with other circular building systems, watertight and vapor tight sealing of both surfaces and joints, diversity of products within circular building systems (e.g. tailored lintels, corner solutions), broad building type (in view of needed performances) and project type application range (e.g. renovation); prefabrication potential, lowering the environmental impact (e.g. especially of connecting components) of existing circular building systems, and more broadly development of new circular building systems based on renewable and regenerative resources and considering open source knowledge and system development to maximize the development and implementation potential of circular systems.

\section{Acknowledgements}

This student research was possible thanks to involved manufacturers.

\section{References}

[1] EC (European Commission), 2011. Roadmap to a resource efficient Europe COM (2011) 571 final (Brussels: European Commission).

[2] Janssens, B. and Verbruggen, M. (2014). Feasibility of upgrading the energy performance of recent massive brick houses. Frontiers of Architectural Research, 3, p. 44-54.

[3] Rovers, R. (2008). Sustainable Housing Projects: Implementing a Conceptual Approach. Amsterdam: Techne Press.

[4] Hegger, M., Fuchs, M., Stark, T. and Zeumer, M. (2008). Energy Manual: Sustainable Architecture. Munich: Edition Detail, Birkhäuser.

[5] Abdalla, G., Maas, G., Huyghe, J. and Oostra, M. (2011). Criticism on environmental assessment tools. IPCBEE, 6, pp. V2-443-V2-445.

[6] WEF (World Economic Forum), 2015. New Vision for Education: Unlocking the Potential of Technology.

[7] Vandenbroucke M. (2016). Using environmental life cycle assessment to inform decision-making for demountable building elements. Doctoral dissertation. Vrije Universiteit Brussel. 